\title{
1-(3-ACETYL PHENYL)-4-(4-ARYLIDENE)-2-PHENYL-1H- IMIDAZOL-5(4H)-ONES: SYNTHESIS, SPECTROSCOPIC CHARACTERIZATION, ANTIMICROBIAL AND MOLECULAR DOCKING STUDIES
}

\author{
Y. Aparna ${ }^{1, \bigotimes}$, D. Sandhya ${ }^{2}$, N. J. P. Subhashini ${ }^{3}$ and L. N. Sharada \\ ${ }^{1}$ Matrusri Engineering College, Saidabad-500059, Hyderabad, Telangana, India \\ ${ }^{2,3,4}$ Department of Chemistry, Osmania University, Hyderabad-500007, Telangana, India \\ ${ }^{\otimes}$ Corresponding Author: aparnayeddala@gmail.com
}

\begin{abstract}
Imidazole rings are privileged moieties in heterocyclic chemistry since they permit functional group and structural modifications to synthesize abundant substituted heterocycles. Thus, the pursuit of exploring many more alterations on imidazole moiety needs to be continued. In the present work an array of the new imidazolone derivatives was prepared as per the expedient method. The moieties were characterized by FTIR, ${ }^{1} \mathrm{HNMR},{ }^{13} \mathrm{CNMR}$ and Mass spectra. Docking was studied to exemplify the binding connections of intended scaffolds with the crystal structure of proteinreductase of Staphylococcusaureus pdbid:4NZ9. The synthesized compounds were tested for antibacterial activity by the agar diffusion method. All the compounds showed potent activity against diverse bacterial species.

Keywords: Oxazolone, Glacial Acetic Acid ,Imidazolone, Molecular Docking, Acetyl phenyl.

RASĀYANJ. Chem., Vol. 14, No.4, 2021
\end{abstract}

\section{INTRODUCTION}

Amid aromatic heterocycles, predominant moieties are five-membered rings. The role and efficacy of heterocycles in organic synthesis surfaced the way to develop precursors for aminoacids, medicinal drugs and other chemical components. For any organic moiety, the effectiveness is measured based on its nontoxic nature, lower dosage and inhibition of microbial growth. The research activity in heterocyclic compounds is intensified on the expansion of biologically active scaffolds, likewise on formulating novel molecules for assessing their potency as drugs, pharmaceuticals, special chemicals and agrochemicals.

In this view, the synthesis of new drugs with a different mode of action is desirable to be explored. Imidazolone-5-ones form a significant class of heterocyclic moieties and are acknowledged to display pharmacological and biological actions such as CNS depressant, antifungal, antihelmentic, anticancer, anticonvulsant, anti-parkinsonian, anti-inflammatory and monoamineoxidase inhibitoryagents ${ }^{1-10}$. Few new disubstituted imidazolones were proved as an anticonvulsant, monoamine oxidase suppressive and succinate dehydrogenase suppressive agents. ${ }^{11,12}$ Imidazoloquinazolinone derivatives which are synthesized from arylidene-2-phenyl-oxaolone derivatives, showed promising Antibacterial activity. ${ }^{13} 2,4,5$-triphenyl-imidazoles are first-rate crucial ligands in Organometallic chemistry as their complexes of $\mathrm{Cu}^{+2}$ displayed anti-E.coli non-pathogenic strain activity. ${ }^{14}$ Thus, the pursuit to explore modifications on imidazole ringdesires to be sustained. The present work comprises of synthesis of novel imidazolinones(6a-j) and is evaluated for their antimicrobial activity. In the direction of elucidating the antibacterial assay, molecular docking studies on staphylococcus aureus were performed.

\section{Material and Methods}

\section{EXPERIMENTAL}

The experimental ingredients that were used are of analytical grade. The compounds there were prepared and checked in open-glass capillaries on Staurt-SMP10 melting point apparatus and were uncorrected. 
RASĀYAN J. Chem.

Vol. 14 | No. 4 |2442-2450| October- December | 2021

The purity of the sample was checked by (TLC)plates DC $0.25 \mathrm{~mm}, 60 \mathrm{~F}_{254}$ coated sheets procured from merck, and spots were envisaged by iodinevapor(or)U.Vlight.Ten novel imidazolones were synthesized from azlactones by reported procedures. ${ }^{15}$ Molecular docking studies were done on Linux operating system using Schrodinger suite 2009. Crystal structure of reductaseprotein of Staphylococcus aureus was downloaded from the protein data bank pdbid:4NZ9. The protein fragments were set by protein groundwork wizard applying OPLS2005 forcefield, and a grid was prepared around the bindingsite by choosing the co-crystallizedligand. Imidazole derivatives were built in Maestro-build-panel and outfitted by Ligprep-module. These were docked in requisite-site using Glide-5.6. Ligand interaction diagrams were obtained using Maestro academic-version and figures were obtained using DS4.0 viewer.

\section{General Procedure for Synthesis 4-benzylidene-2-phenyl-2-oxazoline-5-one (4a-j)}

4-benzylidene-2-phenyl-2-oxazoline-5-ones (4a-j) are prepared by condensation of aromatic aldehydes with benzoyl derivative of glycine in the presence of $\left(\mathrm{CH}_{3} \mathrm{CO}\right)_{2} \mathrm{O}$ and fused anhydrous sodium acetate $0.008 \mathrm{~mol}$ of benzoylglycine(3)prepared by stirring glycine $(0.33 \mathrm{~mol})$ with benzoyl chloride $(0.385 \mathrm{~mol}), 1$ gram of freshly fused sodium acetate and 2-3 $\mathrm{ml}$ of acetic anhydride was added with slow stirring and stirring continued for 30 seconds. Then immediately, $0.008 \mathrm{~mol}$ aromatic aldehyde was added and refluxed on a hot waterbath for about 1 hour. Continuation of the reaction was observed by using TLC.7ml ethanol was added to it and kept overnight, which resulted in the yellow crystalline precipitate, which was purified by washing with cold ethanol followed by boiling water.
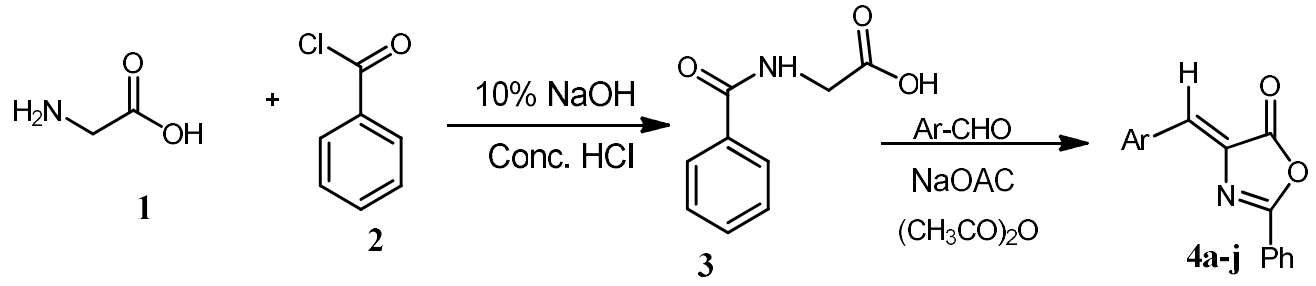

$\mathrm{Ar}=$ phenyl, 2-chloro phenyl, 4-chloro phenyl, 2,4 dichloro phenyl

2,6 dichloropheny,2-hydroxy phenyl,4-hydroxy phenyl,4-nitro phenyl, 3,4,5-trimethoxy phenyl, 2,5-dimethoxy phenyl

Scheme-1: Synthetic Reactions of Oxazolones 4a-j

\section{Synthesis of Novel-1H-imidazole-5(4H)-ones}

$1 \mathrm{~mol}$ of 4-arylidene-2-phenyl-2-oxazoline-5-ones (4a-j) ,1mol of 3-amino acetophenone (5), $15 \mathrm{ml}$ glacial acetic acid and $\mathrm{CH}_{3} \mathrm{COONa}$ were refluxed for $3 \mathrm{hrs}$ at $100^{\circ} \mathrm{C}$ in aRB flask. After checking the continuity of reaction monitored by TLC, the reaction mass was transferred into crumpled ice. The precipitate was dried and run by column chromatography(Scheme-2).

\section{1-(3-acetylphenyl)-4-imidazolderivative(6a)}

FT-IR(KBrcm $\left.{ }^{-1}\right) 3421,2922,1800,1633,1448,1295,1170,877,759,698 \mathrm{~cm}^{-1} .{ }^{1} \mathrm{H}$ NMR (400 MHz, $\left.\mathrm{CDCl}_{3}\right): \delta 8.28(\mathrm{~s}, 1 \mathrm{H}$,aromatic), $8.11(\mathrm{~s}, 1 \mathrm{H}, \mathrm{CH}), 7.68(\mathrm{t}, J=7.81 \mathrm{~Hz}, 2 \mathrm{H}$, aromatic), $7.51(\mathrm{t}, J=7.44 \mathrm{~Hz}$, $2 \mathrm{H}$,aromatic $), 7.42(\mathrm{~d}, J=7.23 \mathrm{~Hz}, 2 \mathrm{H}$, aromatic $), 7.12(\mathrm{~d}, J=7.55 \mathrm{~Hz}, 2 \mathrm{H}$, aromatic $), 6.83 \quad(\mathrm{~d}, J=$ $7.14 \mathrm{~Hz}, 1 \mathrm{H}$,aromatic), $2.36\left(\mathrm{~s}, 3 \mathrm{H}, \mathrm{CH}_{3}\right) \mathrm{ppm} \cdot \mathrm{MS}-\mathrm{ESIm} / \mathrm{z} 368[\mathrm{M}+2 \mathrm{H}]^{+}$.

\section{1-(3-acetylphenyl)-4-(2-chlorobenzylidene)-imidazol-one(6b)}

FT-IR $\left(\mathrm{KBr} \mathrm{cm}^{-1}\right) \quad 3447, \quad 2923, \quad 1789, \quad 1622, \quad 1555, \quad 1488, \quad 1285, \quad 926, \quad 880,755 \mathrm{~cm}^{-1} .{ }^{1} \mathrm{H}$ $\operatorname{NMR}(\delta): 8.15(\mathrm{~s}, 1 \mathrm{H}$,aromatic), $8.10(\mathrm{~s}, 1 \mathrm{H}, \mathrm{CH}), 7.62(\mathrm{t}, J=7.5 \mathrm{~Hz}, 2 \mathrm{H}$,aromatic), $7.53(\mathrm{~m}, 6 \mathrm{H}$,aromatic), 7.42(d, $1 \mathrm{H}$,aromatic), 7.14(d, $J=7.6 \mathrm{~Hz}, 3 \mathrm{H}$,aromatic), 2.38(s, $\left.3 \mathrm{H}, \mathrm{CH}_{3}\right) \mathrm{ppm}$. MS-ESIm/z $401[\mathrm{M}+\mathrm{H}]^{+}$.

\section{1-(3-acetylphenyl)-4-(4-chlorobenzylidene)-1H-imidazolone(6c)}

FT-IR $(\mathrm{KBr} \mathrm{cm}-1) 3449,2920,2849,1784,1634,1550,1289,878,694 \mathrm{~cm}^{-1} .{ }^{1} \mathrm{H}$ NMR : 8.15 (s,1H,aromatic), $8.10 \quad(\mathrm{~s}, 1 \mathrm{H}, \mathrm{CH} \quad), 7.62 \quad(\mathrm{t}, J=7.5 \mathrm{~Hz}, 2 \mathrm{H}$,aromatic), $7.53 \quad$ (m, 6H,aromatic), 7.42(d,2H,aromatic), 7.22(d,J=7.6 Hz,2H,aromatic), 2.38(s,3H, $\left.\mathrm{CH}_{3}\right)$ ppm.MS-ESIm/z $401[\mathrm{M}+\mathrm{H}]^{+}$. 
RASĀYAN J. Chem.

Vol. 14 | No. 4 |2442-2450| October- December | 2021

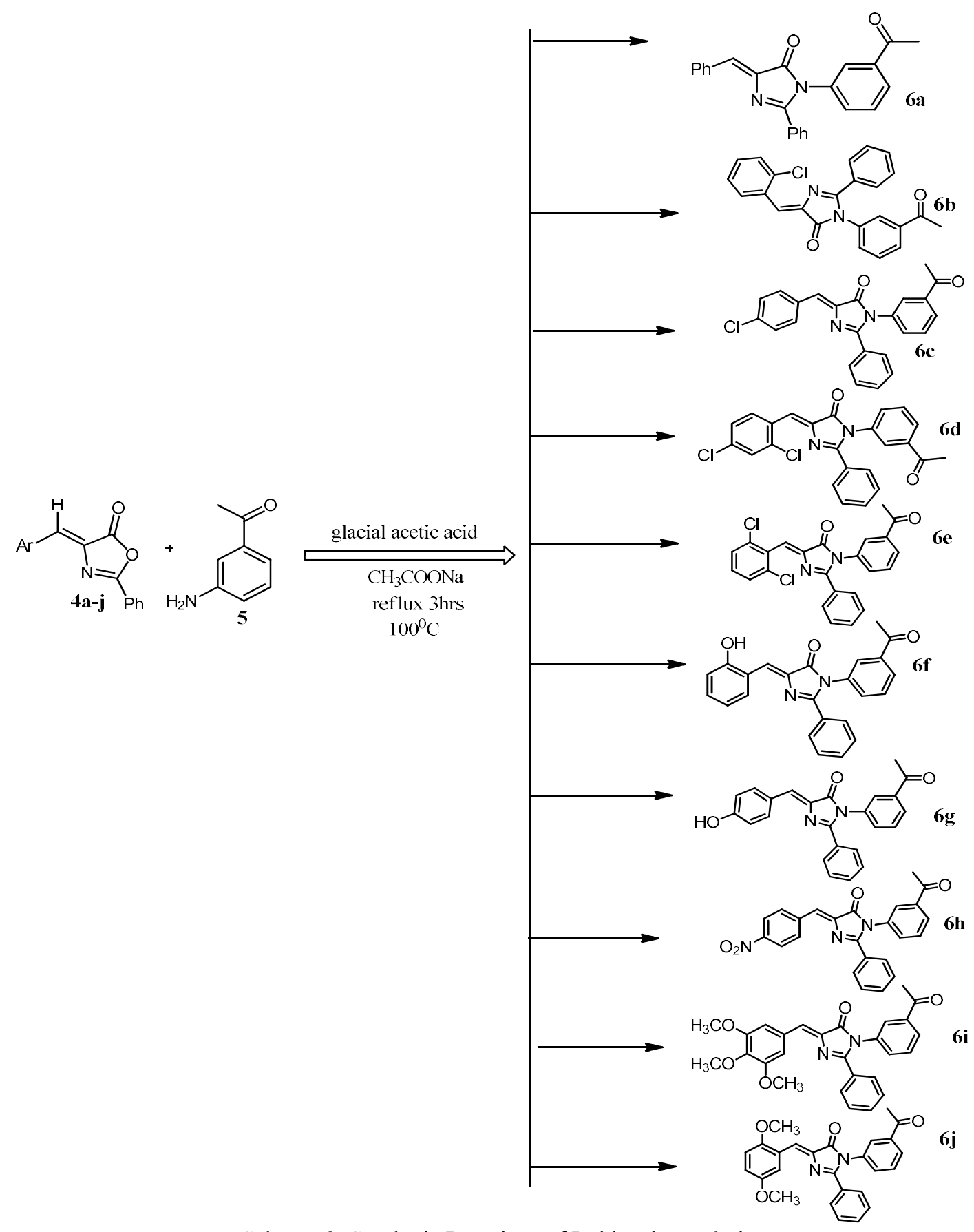

Scheme-2: Synthetic Reactions of Imidazolones 6a-j

1-(3-acetylphenyl)-4-(2,4-benzylidenedichloro)-2-phenyl-imidazol-5(4H)-one(6d)

FT-IR (KBr cm$\left.{ }^{-1}\right)$ 3557, 3313, 2923, 1644, 1563, 1267, 828, 780, $536 \mathrm{~cm}^{-1}$;HNMR :8.15 ( $\mathrm{s}, 1 \mathrm{H}$,aromatic), $8.10(\mathrm{~s}, 1 \mathrm{H}, \mathrm{CH}), 7.62(\mathrm{t}, J=7.36 \mathrm{~Hz}, 2 \mathrm{H}$, aromatic), $7.53(\mathrm{~m}, 5 \mathrm{H}$, aromatic), $7.42(\mathrm{~d}, 3 \mathrm{H}$, aromatic), 7.12(d, $J=7.52 \mathrm{~Hz}, 1 \mathrm{H}$, aromatic), $2.38\left(\mathrm{~s}, 3 \mathrm{H}, \mathrm{CH}_{3}\right) \mathrm{ppm} \cdot \mathrm{MS}-\mathrm{ESIm} / \mathrm{z} 437[\mathrm{M}+\mathrm{H}]^{+}$.

\section{4-(2,6-dichlorobenzylidene)-1-(3-acetylphenyl)-2-phenyl-1H-imidazol-5(4H)-one(6e)}

FT-IR $\left(\mathrm{KBr} \mathrm{cm}^{-1}\right) 3300,2927,1707,1619,1457,1151,955,777,637 \mathrm{~cm}-1 .{ }^{1} \mathrm{H}$ NMR $\delta 8.15(\mathrm{~s}, 1 \mathrm{H}$, aromatic), $8.10(\mathrm{~s}, 1 \mathrm{H}, \mathrm{CH}), 7.62(\mathrm{t}, J=7.42 \mathrm{~Hz}, 2 \mathrm{H}$, aromatic), $7.51(\mathrm{~m}, 5 \mathrm{H}$, aromatic), $7.42(\mathrm{~d}, J=7.61$ $\mathrm{Hz}, 2 \mathrm{H}$, aromatic), 7.11 (d, $J=7.52 \mathrm{~Hz}, 2 \mathrm{H}$,aromatic), $2.38\left(\mathrm{~s}, 3 \mathrm{H}, \mathrm{CH}_{3}\right) \mathrm{ppm} . \mathrm{MS}-\mathrm{ESIm} / \mathrm{z} 437$ [M + H]+. 
RASĀYAN J. Chem.

Vol. 14 | No. 4 |2442-2450| October- December | 2021

4-(2-hydroxybenzylidene)-1-(3- acetylphenyl)-2-phenyl-1H-imidazol-5(4H)-one(6f)

FT- IR $\left(\mathrm{KBr} \mathrm{cm}^{-1}\right) 3499,2923,2853,1643,1539,1429,1397,1099,712,668 \mathrm{~cm}^{-1} .{ }^{1} \mathrm{H}$ NMR $\delta 8.28$ $(\mathrm{s}, 1 \mathrm{H}, \mathrm{CH}), 8.10(\mathrm{~s}, 1 \mathrm{H}$,aromatic $), 7.64(\mathrm{t}, J=7.42 \mathrm{~Hz}, 2 \mathrm{H}$,aromatic $), 7.61(\mathrm{t}, J=7.53 \mathrm{~Hz}, 2 \mathrm{H}$, aromatic), $7.51(\mathrm{~m}, 3 \mathrm{H}$, aromatic), $7.42(\mathrm{~d}, J=7.72 \mathrm{~Hz}, 2 \mathrm{H}$, aromatic), $7.11(\mathrm{~d}, J=7.43 \mathrm{~Hz}, 1 \mathrm{H}$, aromatic), $6.82(\mathrm{~d}, J=7.10 \mathrm{~Hz}, 1 \mathrm{H}$, aromatic), $6.63(\mathrm{~d}, J=7.33 \mathrm{~Hz}, 1 \mathrm{H}$, aromatic), $4.82(\mathrm{~s}, 1 \mathrm{H}, \mathrm{OH}), 2.41(\mathrm{~s}, 3 \mathrm{H}$, $\left.\mathrm{CH}_{3}\right) \mathrm{ppm} . \mathrm{MS}-\mathrm{ESIm} / \mathrm{z} 383[\mathrm{M}+\mathrm{H}]^{+}$.

\section{4-(4-hydroxybenzylidene)-1-(3-acetylphenyl)-)-2-phenyl-1H-imidazol-5(4H)-one(6g)}

FT-IR $\left(\mathrm{KBr} \mathrm{cm}^{-1}\right) 3419,2924,1629,1550,1321,891,750,676 \mathrm{~cm}^{-1} .{ }^{1} \mathrm{H}$ NMR: 8.11 (s, 1H, aromatic), $7.86(\mathrm{~s}, 1 \mathrm{H}, \mathrm{CH}), 7.61(\mathrm{t}, J=7.44 \mathrm{~Hz}, 2 \mathrm{H}$, aromatic), 7.51 (m,3H,aromatic), 7.48 (m,5H,aromatic), 6.82 $\left(\mathrm{d}, J=7.10 \mathrm{~Hz}, 2 \mathrm{H}\right.$,aromatic), $4.87(\mathrm{~s}, 1 \mathrm{H}, \mathrm{OH}), 2.36\left(\mathrm{~s}, 3 \mathrm{H}, \mathrm{CH}_{3}\right) \mathrm{ppm} . \mathrm{MS}-\mathrm{ESIm} / \mathrm{z} 383\left[\mathrm{M}^{+}\right]^{+}$.

\section{1-(3-acetylphenyl)-4-(4-nitrobenzylidene)-2-phenyl-1H-imidazol-one(6h)}

FT-IR $\left(\mathrm{KBr} \mathrm{cm}^{-1}\right) 3010,2925,1665,1622,1548,1444,1307,1053,703,678 \mathrm{~cm}^{-1} .{ }^{1} \mathrm{H}$ NMR $\delta: 8.25$ (t, $J=$ $7.46 \mathrm{~Hz}, 3 \mathrm{H}$, aromatic), $8.09(\mathrm{~d}, J=7.22 \mathrm{~Hz}, 2 \mathrm{H}$, aromatic), $7.93(\mathrm{~s}, 1 \mathrm{H}, \mathrm{CH}), 7.59(\mathrm{t}, J=7.44$ $\mathrm{Hz}, 2 \mathrm{H}$,aromatic), $7.51(\mathrm{~m}, 3 \mathrm{H}$, aromatic), $7.41(\mathrm{~d}, J=7.64 \mathrm{~Hz}, 2 \mathrm{H}$,aromatic), $7.29(\mathrm{t}, J=7.10$ $\mathrm{Hz}, 1 \mathrm{H}$,aromatic), 2.39(s,3H, $\left.\mathrm{CH}_{3}\right) \mathrm{ppm}$. MS-ESIm/z $412[\mathrm{M}+\mathrm{H}]^{+}$.

\section{1-(3-acetylphenyl)-2-phenyl-4-(-3,4,5-trimethoxybenzylidene)-imidazol-5(4H)-one(6i)}

FT-IR $\left(\mathrm{KBrcm}^{-1}\right)$ 3449, 2920, 1784, 1638, 1550, 1289, 1164, 817, $694 \mathrm{~cm}^{-1} .{ }^{1} \mathrm{H}$ NMR: $8.20(\mathrm{~s}, 1 \mathrm{H}$, aromatic), $8.09(\mathrm{~s}, 1 \mathrm{H}, \mathrm{CH}), 7.61(\mathrm{t}, J=7.48 \mathrm{~Hz}, 2 \mathrm{H}$, aromatic), 7.51 (m,6H,aromatic), 6.45 (s,2H,aromatic), $3.82\left(\mathrm{~s}, 9 \mathrm{H}, \mathrm{OCH}_{3}\right), 2.46\left(\mathrm{~s}, 3 \mathrm{H}, \mathrm{CH}_{3}\right) \mathrm{ppm} . \mathrm{MS}-\mathrm{ESIm} / \mathrm{z} 457[\mathrm{M}+\mathrm{H}]^{+}$.

\section{1-(-3-acetylphenyl)-4-(2,5-dimethoxybenzylidene)-2-phenyl-imidazolone(6j)}

FT-IR $\left(\mathrm{KBrcm}^{-1}\right)$ 3436, 2925, 1787, 1638, 1599, 1555, 1451, 1332, 1188, 882, $696 \mathrm{~cm}^{-1} .{ }^{1} \mathrm{H}$ NMR $\delta .21$ (s,1H,aromatic), $8.10(\mathrm{~s}, 1 \mathrm{H}, \mathrm{CH}), 7.60(\mathrm{t}, J=7.52 \mathrm{~Hz}, 2 \mathrm{H}$, aromatic), 7.50 (m,6H,aromatic), 6.52( d, $J=$ $7.77 \mathrm{~Hz}, 2 \mathrm{H}$,aromatic), 6.49 (s,1H,aromatic), $3.80\left(\mathrm{~s}, 6 \mathrm{H}, \mathrm{OCH}_{3}\right), 2.48\left(\mathrm{~s}, 3 \mathrm{H}, \mathrm{CH}_{3}\right)$ ppm. MS-ESIm/z 427 $[\mathrm{M}+\mathrm{H}]^{+}$.

\section{Detection Method}

The prepared derivatives were checked in open glasscapillaries on Staurt SMP10 meltingpointinstrument and were uncorrected. TLC was used to identify the purity of the sample, and the single spot was visualized under U.V light. The IR spectra were obtained with IR AFFINITY-1 FTIR Shimadzu spectrometer. $\mathrm{H}^{1} \mathrm{NMR}$ spectra $(\mathrm{ppm})$ were noted in $\mathrm{CDCl}_{3}$ and $\mathrm{DMSO}-\mathrm{d}_{6}$ on a varianmercury $300 \mathrm{MHz}$ spectrometer. $\mathrm{C}^{13} \mathrm{NMR}$ spectra have been recorded in $\mathrm{CDCl}_{3}$ and DMSO- $\mathrm{d}_{6}$ on a Bruker Avance II400 spectrometer at $400 \mathrm{MHz}$. And the mass spectra have been recorded on a Shimadzu. All derivatives were regularly checked by TLC by means of $\mathrm{CHCl}_{3}: \mathrm{CH}_{3} \mathrm{OH}$ (9:1) solvent system and checked under UV light.

\section{Analytical Discussion}

As a representative case, the spectral analysis of (6a) is considered. In the IR spectrum of (6a), the stretching frequency at $3421 \mathrm{~cm}^{-1}$ and $2922 \mathrm{~cm}$-1indicates absorption due to the aliphatic $(\mathrm{C}-\mathrm{H})$ group. The characteristic $(\mathrm{C}=\mathrm{N})$ absorption was observed at $1448 \mathrm{~cm}^{-1}$ and $(\mathrm{C}-\mathrm{N})$ group absorption $1295 \mathrm{~cm}^{-1}$. The ${ }^{1} \mathrm{HNMR}$ spectrum $\left(400 \mathrm{MHz}, \mathrm{CDCl}_{3}\right.$, ) (6a) showed multiplet between $\delta$ 7.12-8.11 due to aromatic protons and $\delta 8.28$ due to arylidene proton. ESI-MS mass spectra of derivative $(6 a) \mathrm{m} / \mathrm{z} 368(\mathrm{M}+2 \mathrm{H})^{+}$ appeared as base peak.

\section{Proposed Mechanism}

\section{RESULTS AND DISCUSSION}

The mechanism involves Erlenmeyer plochl reaction which involves perkin condensation where acetic anhydride reacts with substituted aromatic aldehydes to undergo cyclization and 
RASĀYAN J. Chem.

Vol. 14 | No. 4 |2442-2450| October- December | 2021

condensation reaction in presence of weak base.Intermolecular acetylation reaction occurs via alkoxide anhydride intermediate in this mechanism.

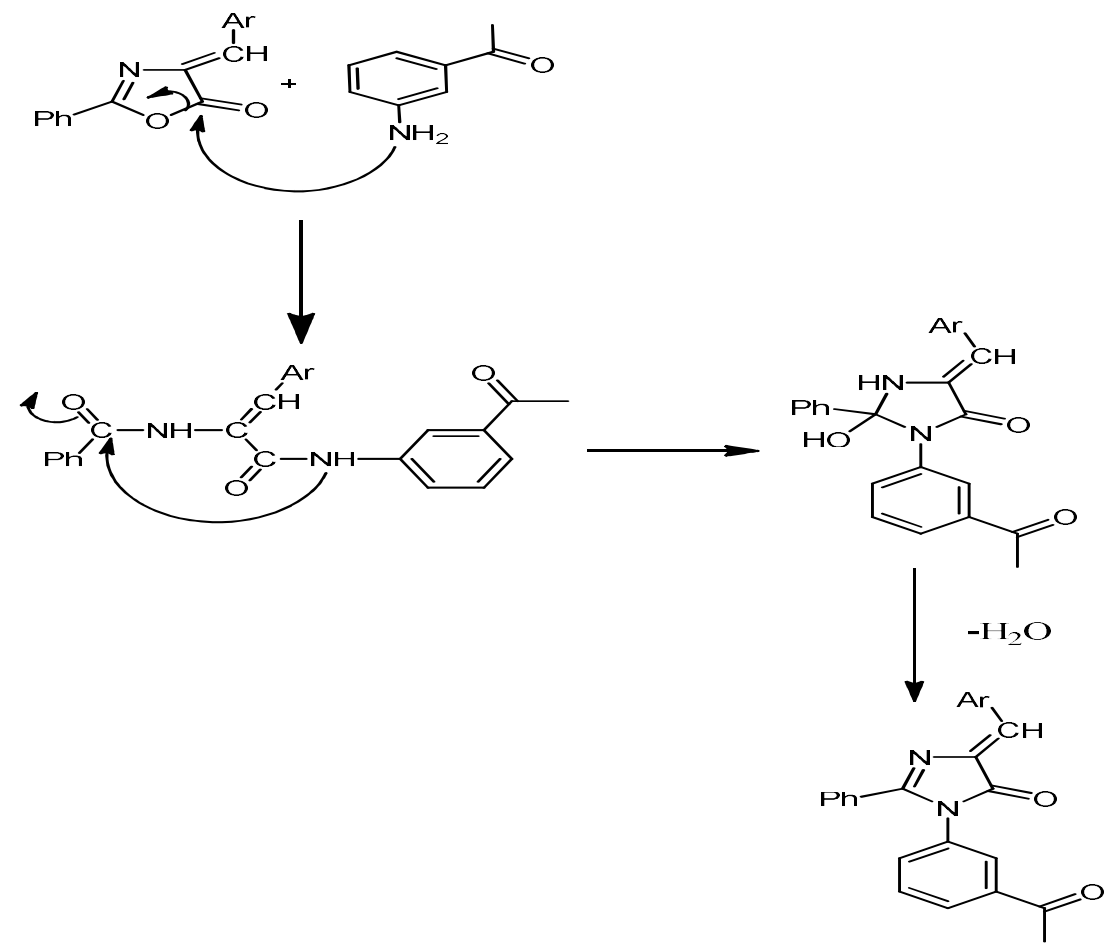

Table-1: Physical Characterization of Compounds (6a-j)

\begin{tabular}{|c|c|c|c|c|c|}
\hline Compound & Systemic Name & $\begin{array}{l}\text { Molecular } \\
\text { Formula }\end{array}$ & $\begin{array}{c}\text { Yield } \\
\%\end{array}$ & $\begin{array}{l}\text { M.P } \\
\left({ }^{0} \mathrm{C}\right)\end{array}$ & $\begin{array}{c}\text { Color of } \\
\text { Compound }\end{array}$ \\
\hline $6 a$ & $\begin{array}{l}\text { 1-(3-acetyl phenyl)-4- } \\
\text { benzylidene-2-phenyl-1H- } \\
\text { imidazol -5(4H)-one }\end{array}$ & $\mathrm{C}_{24} \mathrm{H}_{18} \mathrm{~N}_{2} \mathrm{O}_{2}$ & 80 & $232^{\circ} \mathrm{C}$ & Orange solid \\
\hline $6 b$ & $\begin{array}{c}\text { 1-(-3-acetylphenyl)-4-(2- } \\
\text { chlorobenzylidene)-2-phenyl- } \\
\text { 1H-imidazol-5(4H)-one }\end{array}$ & $\mathrm{C}_{24} \mathrm{H}_{17} \mathrm{ClN}_{2} \mathrm{O}_{2}$ & 82 & $195^{\circ} \mathrm{C}$ & $\begin{array}{l}\text { Yellowish } \\
\text { Orange solid }\end{array}$ \\
\hline $6 c$ & $\begin{array}{l}\text { 1-(3-acetylphenyl )-4-(4- } \\
\text { chlorobenzylidene)-2-phenyl- } \\
\text { 1H-imidazol -5(4H)-one }\end{array}$ & $\mathrm{C}_{24} \mathrm{H}_{17} \mathrm{ClN}_{2} \mathrm{O}_{2}$ & 85 & $256^{\circ} \mathrm{C}$ & $\begin{array}{l}\text { Yellowish } \\
\text { Orange solid }\end{array}$ \\
\hline $6 \mathrm{~d}$ & $\begin{array}{l}\text { 1-(3 -acetylphenyl)-4- } \\
\text { (2,4dichlorobenzylidene)-2- } \\
\text { phenyl-imidazol-5(4H)-one }\end{array}$ & $\mathrm{C}_{24} \mathrm{H}_{16} \mathrm{Cl}_{2} \mathrm{~N}_{2} \mathrm{O}_{2}$ & 78 & $278^{\circ} \mathrm{C}$ & $\begin{array}{l}\text { light orange } \\
\text { solid }\end{array}$ \\
\hline $6 e$ & $\begin{array}{c}\text { 1-( }-3 \text {-acetylphenyl-)-4- } \\
\text { (2,6dichlorobenzylidene)-2- } \\
\text { phenyl-imidazolone }\end{array}$ & $\mathrm{C}_{24} \mathrm{H}_{16} \mathrm{Cl}_{2} \mathrm{~N}_{2} \mathrm{O}_{2}$ & 82 & $280^{\circ} \mathrm{C}$ & $\begin{array}{c}\text { Yellowish } \\
\text { orange solid }\end{array}$ \\
\hline
\end{tabular}


RASĀYAN J. Chem.

Vol. 14 | No. 4 |2442-2450| October-December | 2021

\begin{tabular}{c|c|c|c|c|c}
\hline $6 \mathrm{f}$ & $\begin{array}{c}1 \text {-(3-acetylphenyl) -4-(2- } \\
\text { hydroxybenzylidene)-2- } \\
\text { phenyl-1H-imidazol-5-one }\end{array}$ & $\mathrm{C}_{24} \mathrm{H}_{18} \mathrm{~N}_{2} \mathrm{O}_{3}$ & 80 & $267{ }^{\circ} \mathrm{C}$ & $\begin{array}{c}\text { Yellowish } \\
\text { orange solid }\end{array}$ \\
\hline $6 \mathrm{~g}$ & $\begin{array}{c}\text { 1-(3-acetyl phenyl )-4-(4- } \\
\text { hydroxy benzylidene)-2- } \\
\text { phenyl-1H-imidazol-(4H)-one }\end{array}$ & $\mathrm{C}_{24} \mathrm{H}_{18} \mathrm{~N}_{2} \mathrm{O}_{3}$ & 83 & $273^{\circ} \mathrm{C}$ & $\begin{array}{c}\text { Yellow color } \\
\text { solid }\end{array}$ \\
\hline $6 \mathrm{~h}$ & $\begin{array}{c}4-(4-\text { nitrobenzylidene)-1-(3- } \\
\text { acetyl phenyl )-2-phenyl- } \\
\text { imidazol-5(4H)-one }\end{array}$ & $\mathrm{C}_{24} \mathrm{H}_{17} \mathrm{~N}_{3} \mathrm{O}_{4}$ & 84 & $241^{\circ} \mathrm{C}$ & $\begin{array}{c}\text { Yellow color } \\
\text { solid }\end{array}$ \\
\hline $6 \mathrm{i}$ & $\begin{array}{c}4-(3,4,5- \\
\text { trimethoxybenzylidene)-1-(3- } \\
\text { acetylphenyl)-2-phenyl-1H- } \\
\text { imidazolone }\end{array}$ & $\mathrm{C}_{27} \mathrm{H}_{24} \mathrm{~N}_{2} \mathrm{O}_{5}$ & 81 & $239^{\circ} \mathrm{C}$ & $\begin{array}{c}\text { Yellow color } \\
\text { solid }\end{array}$ \\
\hline $6 \mathrm{j}$ & $\begin{array}{c}2,5 \text { dimethoxybenzylidene)-1- } \\
\text { (3-acetylphenyl)-4-(2-phenyl- } \\
1 \mathrm{H}-\text {-imidazol-5(4H)-one }\end{array}$ & $\mathrm{C}_{26} \mathrm{H}_{22} \mathrm{~N}_{2} \mathrm{O}_{4}$ & 86 & $272^{\circ} \mathrm{C}$ & $\begin{array}{c}\text { Yellow color } \\
\text { solid }\end{array}$ \\
\hline & & & & \\
\hline
\end{tabular}

\section{Antibacterial Assay}

The antibacterial assay of synthesized moieties were checked against gram positive bacteria $B$. subtilis and $S$. aureus and two gramnegative bacteria $E$. coli and $S$. typhi. A stock solution of $2 \mathrm{mg} / \mathrm{ml}$ was prepared.Wells were made in the agar plate using a borer. The sample solution is pipetted into the wells. ${ }^{16}$ And the plates were incubated for around 24hrs. Then the region ofrestrained and recorded as shown in Table-2. All compounds exhibited moderate to potent activity against gram(+) and gram(-) bacteria.

Table-2: Antibacterial Activity of 4-(4-arylidene)-1-(3-acetyl phenyl)-2-phenyl-1H-imidazol-5(4H)-ones(6a-j)

\begin{tabular}{c|c|c|c|c}
\hline \multirow{2}{*}{ Compound } & \multicolumn{4}{|c}{ Zone of inhibition(mm) in $2 \mu \mathrm{g} / \mathrm{ml}$ concentration } \\
\cline { 2 - 5 } & $\begin{array}{c}\text { gram } \\
\text {-ve }\end{array}$ & $\begin{array}{c}\text { gram } \\
-v e\end{array}$ & gram + ve & $\begin{array}{c}\text { gram } \\
+v e\end{array}$ \\
\hline & E.coli & $\begin{array}{c}P . \\
\text { aeruginosa }\end{array}$ & B.subtilis & S. aureus \\
\hline $6 \mathrm{a}$ & 12 & 14 & 12 & 10 \\
\hline $6 \mathrm{~b}$ & 12 & 11 & 13 & 14 \\
\hline $6 \mathrm{c}$ & 13 & 14 & 12 & 13 \\
\hline $6 \mathrm{~d}$ & 13 & 12 & 14 & 13 \\
\hline $6 \mathrm{e}$ & 12 & 8 & 14 & 12 \\
\hline $6 \mathrm{f}$ & 13 & 14 & 10 & 14 \\
\hline $6 \mathrm{~g}$ & 12 & 15 & 13 & 18 \\
\hline $6 \mathrm{~h}$ & 10 & 12 & 11 & 13 \\
\hline $6 \mathrm{i}$ & 9 & 11 & 12 & 12 \\
\hline $6 \mathrm{j}$ & 11 & 13 & 14 & 14 \\
\hline
\end{tabular}

\section{Molecular Docking Studies}

Imidazole derivatives have accomplished much contemplation for their anti-microbial activity. Bacterial ENRs are believed to be fineexplicit targets for antibacterial agents developmentand are one of the target proteins that are inhibited by imidazole derivatives. ${ }^{17,18}$ In order to explicate the mode of interaction and binding of synthesized imidazole derivatives, molecular docking studieswere carried out on X-ray crystal structure of FabI of Staphylococcus aureus,pdbid:4NZ9 found from databank (www.rcsb.org). ${ }^{19-22}$ The docking results are provided in Table-3. The highest dock score of $-10.318 \mathrm{kcal} / \mathrm{mol}$ and least dock score of $-9.014 \mathrm{kcal} / \mathrm{mol}$ were obtained for molecules $6 \mathrm{a}$ and $6 \mathrm{~g}$, respectively. All molecules showed a good 
RASĀYAN J. Chem.

Vol. 14 | No. 4 |2442-2450| October- December | 2021

dock score, indicating that all molecules are good inhibitors of enoylacylcarrier protein reductase (Fab I). Dock pose of molecule6g in the active location of Fab I is shown in Fig.-1 and hydrophobic interaction of the molecules with active site amino acid residues is visible in Fig.-2.The hydrogen bonding interaction of ligand is shown in Fig.-3. The molecule showed 2 H-bond interactions with aminoacid residue Asn156 and Ser197; the other major interaction with the protein was of hydrophobic nature with amino acid residues Met 99, Tyr147, Tyr157, Met160, Pro 192 and Ile207 in Fig.-3.

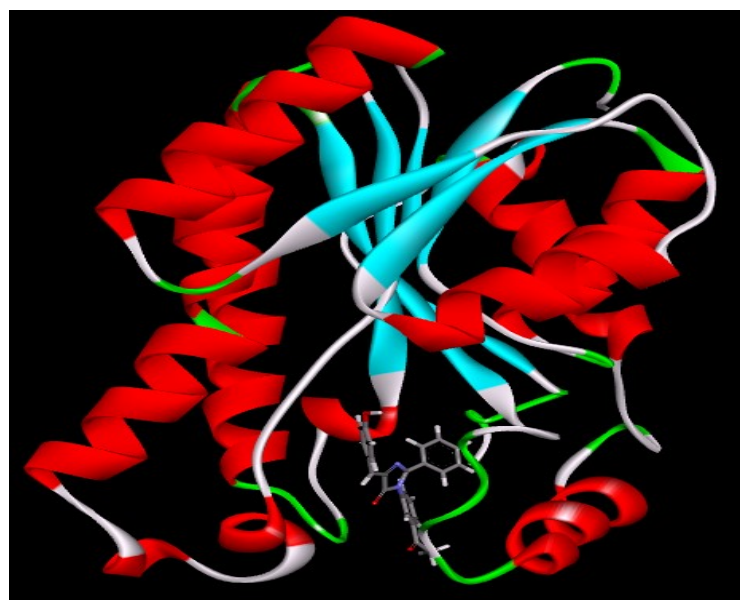

Fig.-1: Dock Pose of Molecule 6g in the Active Site of FABI

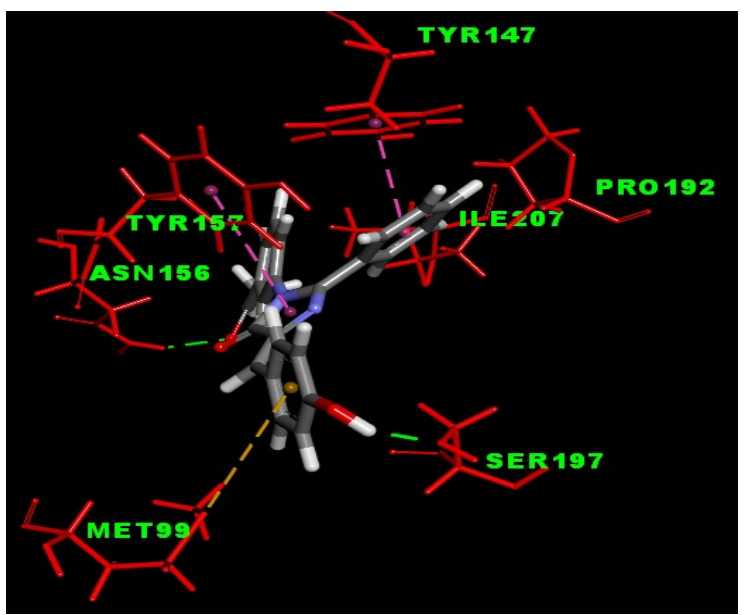

Fig.-2: Hydrophobic Interaction of the Molecules with Dynamic Site Amino Acid Residues

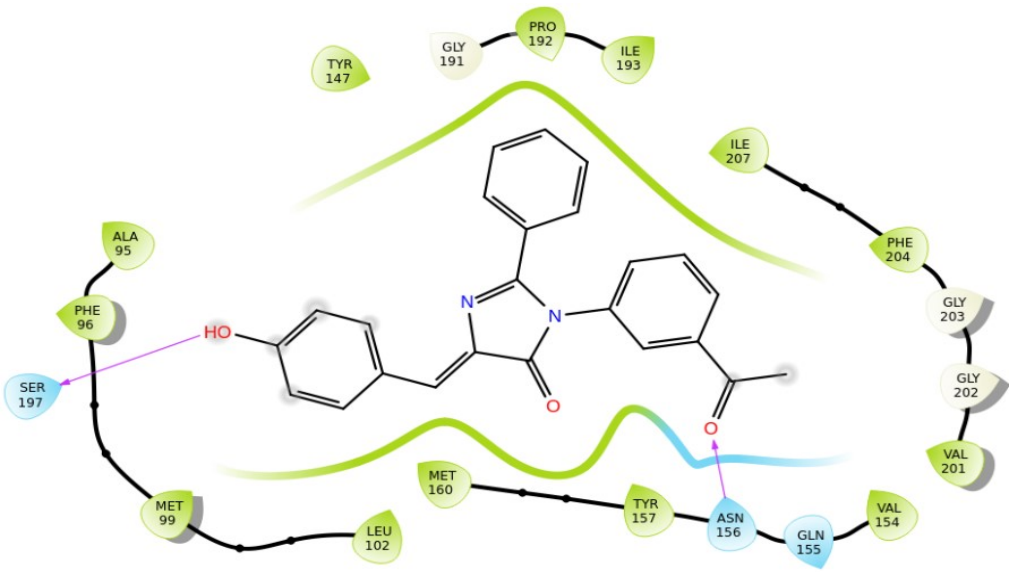

Fig.-3: Ligand Interaction Diagram representing the Hydrogen Bond Interaction. 
RASĀYAN J. Chem.

Vol. 14 | No. 4 |2442-2450| October- December | 2021

Table-3: Dockscore of imidazole derivatives docked into the active site of FabI of S. aureus using extra precision (XP) docking protocol of Glide 5.6.

\begin{tabular}{c|c|c}
\hline S.No. & Entry & Dockscore $(\mathrm{kcal} / \mathrm{mol})$ \\
\hline 1. & $6 \mathrm{a}$ & -10.318 \\
\hline 2. & $6 \mathrm{~b}$ & -9.021 \\
\hline 3. & $6 \mathrm{c}$ & -9.648 \\
\hline 4. & $6 \mathrm{~d}$ & -9.781 \\
\hline 5. & $6 \mathrm{e}$ & -9.159 \\
\hline 6. & $6 \mathrm{f}$ & -9.488 \\
\hline 7. & $6 \mathrm{~g}$ & -9.014 \\
\hline 8. & $6 \mathrm{~h}$ & -9.603 \\
\hline 9. & $6 \mathrm{i}$ & -9.121 \\
\hline 10. & $6 \mathrm{j}$ & -9.511 \\
\hline
\end{tabular}

CONCLUSION

The synthesized novel imidazole scaffolds exhibited wide spectrum antibacterial activity.Compounds $\mathbf{6 a}, \mathbf{6 c}, \mathbf{6 d}, \mathbf{6 g}$ and $6 \mathrm{hdisplayed} \mathrm{potent} \mathrm{activity} \mathrm{against} \mathrm{gram+ve} \mathrm{and} \mathrm{gram} \mathrm{-vebacteria.} \mathrm{Molecular} \mathrm{docking}$ studies with bacterial host DNA inferred confirmative outcomes for the binding affinity of derivatives in accord with experimental data. All the derivatives have shown moderate to good docking scores indicating that they are inhibitors of enoyl acyl carrierprotein reductase. Among all the entries, 6 a showed the least dock score of $-9.014 \mathrm{kcal} / \mathrm{mol}$ and $\mathbf{6 g}$ maximum dock score of $-10.318 \mathrm{kcal} / \mathrm{mol}$. The twang on docking is to computationally conjure upthe molecular recognition process.

\section{ACKNOWLEDGEMENT}

The authors are grateful to the HOD, Dept.of Chemistry,Osmania University, Hyderabad for providing lab facilities and thanks to Management, Matrusri Engineering College, Saidabad, for supporting research work and also thanks to the analytical team, CFRD, Osmania University and IICT, Hyderabad for providing spectral data.

\section{REFERENCES}

1. Prasad Pullagura, M. Krishna, S. Avdhut Kanvinde, S. Raja, International Journal of Pharmacy and Pharmaceutical Sciences, 8(12), 22(2016), https://doi.org/10.22159/ijpps.2016v8i12.14949

2. D. Huang, F. Qiu, Z. Zhang, L. Shi, C. Cao, S. Ke, Journal of Heterocyclic Chemistry, 56(9), 2494(2019), https://doi.org/10.1002/jhet.3639

3. P. Köhler, International Journal for Parasitology, 31(4), 336(2001), https://doi.org/10.1016/S00207519(01)00131-X

4. A. EI Rashedy, H. Y. Aboul-Enein, Mini Reviews in Medicinal Chemistry, 13(3), 399-(2013), https://doi.org/10.2174/138955713804999847

5. P. M. Vasil'ev, K. Y. Kalitin, A. A. Spasov, O. Y. Grechko, V. V. Poroikov, D. A. Filimonov, V. Anisimova, Pharmaceutical Chemistry Journal, 50(12), 775(2017), https://doi.org/10.1007/s11094$\underline{017-1530-6}$

6. S. P. Singh, S. S. Parmar, B. R. Pandey, Journal of Heterocyclic Chemistry, 14(6), 1093(1977), https://doi.org/10.1021/jm00260a027

7. N. Anastassova, D. Aluani, A. Kostadinov, M. Rangelov, N.Todorova, N. Hristova-Avakumova, D.Yancheva, Neural Regeneration Research, 16(11), 2299(2021), https://doi.org/10.4103/1673$\underline{5374.309843}$

8. S. N. A. Bukhari, A. Lauro, G. Jantan, I. Fei, C. Chee, M. W. Amjad, G. Bifulco, N. A. Rahman, Future Medicinal Chemistry, 8(16), 1953(2016), https://doi.org/10.4155/fmc-2016-0062

9. G. Chen, Z. Liu, Y. Zhang, X. Shan, L. Jiang, Y. Zhao, G. Liang, ACS Medicinal Chemistry Letters, 4(1), 69(2013), https://doi.org/10.1021/ml300282t

10. O. D. Can, D. Osmaniye, U. D. Özkay, B. N. Sağlık, S. Levent, S. Ilgın, Z. A. Kaplancıklı, European Journal of Medicinal Chemistry, 131, 92(2017), https://doi.org/10.1016/j.ejmech.2017.03.009 
RASĀYAN J. Chem.

Vol. 14 | No. 4 |2442-2450| October- December | 2021

11. D. van den Berg, K. R. Zoellner, M. O. Ogunremi, S. F. Malan, G. Terre 'Blanche, N. Castagnoli Jr, J. P. Pitzer, Bioorganic \& Medicinal Chemistry, 15(11), 3692(2007), https://doi.org/10.1016/j.bmc.2007.03.046

12. Y. H. Ye, L. Ma, Z. C. Dai, Y. Xiao, Y. Y. Zhang, D. D. Li, H. L. Zhu, Journal of Agricultural and Food Chemistry, 62(18), 4063(2014), https://doi.org/10.1021/jf405437k

13. Solankee, Anjani, Sejal Solankee, and Ghanshyam Patel, Rasayan Journal of Chemistry, 1, 228(2008).

14. T. H. Sucipto, W. Setyarini , F. Martak, D. Raharjo, Rasayan Journal of Chemistry, 14(2), 672(2021), http://dx.doi.org/10.31788/RJC.2021.1425826

15. S. Furniss, A. J. Hannaford, Furniss, Tatchell, A. R. Vogel's Text Book of Practical Organic Chemistry, $5^{\text {th }}$ edition, Longman Group, Essex, UK(1989).

16. S. Magali, S. Mata-Essaying, C. Hartung de Capriles, International Journal of Infectious Diseases, 8 , 39(2004).

17. L. L. Ling, J. Xian, S. Ali, B. Geng, J. Fan, D. M. Mills, D. T. Moir, Antimicrobial Agents and Chemotherapy, 48(5), 1541(2004), https://doi.org/10.1128/AAC.48.5.1541-1547.2004

18. A. E. Cho, V. Guallar, B. J. Berne, R. Friesner, Journal of Computational Chemistry, 26(9), 915(2005), https://doi.org/10.1002/jcc.20222

19. H. J. Böhm, Journal of Computer-aided Molecular Design, 8(3), 243(1994), https://doi.org/10.1007/BF00126743

20. G. Klebe, T. Mietzner, Journal of Computer-Aided Molecular Design, 8(5), 583(1994), https://doi.org/10.1007/BF00123667

21. R. A. Friesner, J. L. Banks, R. B. Murphy, T. A. Halgren, J. J. Klicic, D. T. Mainz, P. S. Shenkin, Journal of Medicinal Chemistry, 47(7), 1739(2004), https://doi.org/10.1021/jm0306430

22. T. A. Halgren, R. B. Murphy, R. A. Friesner, H. S. Beard, L. L. Frye, W. T. Pollard, J. L. Banks, Journal of Medicinal Chemistry, 47(7), 1750(2004), https://doi.org/10.1021/jm030644s

23. Y. Aparna, L. N. Sharada, N. J. P. Subhashini, S. Sreekanth, Russian Journal of General Chemistry, 89(6), 1202(2019), https://doi.org/10.1134/S1070363219060161

[RJC6520/2021] 